J. Clin. Chem. Clin. Biochem.

Vol. 18, 1980, pp. 567-570

\title{
Quantitative Bestimmung der o-Phthaldialdehyd-Derivate von Noradrenalin, Dopamin und Normetanephrin aus dem Urin mit einem Aminosäureanalysator
}

\author{
Von $K$. Olek, S. Uhlhaas und $P$. Wardenbach \\ Institut für Humangenetik der Universität Bonn
}

(Eingegangen am 6. November 1979/27. März 1980)

\begin{abstract}
Zusammenfassung: Mit einem handelsüblichen Aminosäureanalysator werden Noradrenalin, Dopamin und Normetanephrin aus dem Urin an einem Kationenaustauscher (DC 6A Durrum) säulenchromatographisch getrennt. Nach der Auftrennung erfolgt die Derivatisierung mit $o$-Phthaldialdehyd mit dem in der Aminosäurechromatographie üblichen Pumpsystem. Die quantitative Messung wird mit einem Fluorimeter durchgeführt. Zur Vorreinigung werden Fertigsäulen verwendet (Bio-Rad).
\end{abstract}

Quantitative determination of the o-phthaldialhehyde derivatives of noradrenaline, dopamine and normetadrenaline in urine with an amino acid analyzer

Summary: Noradrenaline, dopamine and normetadrenaline in urine are separated by a cation-exchange resin (DC 6A, Durrum) and a commercially available amino acid analyzer. After separation, the $o$-phthalaldehyde derivatives of the compounds are formed by the usual pumping system and are quantified with a fluorimeter. Pre-packed ion-exchange columns (Bio-Rad, Munich) are used for purification and fractionation of the urine samples.

\section{Einführung}

Methoden zur quantitativen Bestimmung biogener Amine in biologischen Flüssigkeiten erfordern eine große Nachweisempfindlichkeit und Spezifität. Letzteres gilt vor allem für Analyșen aus dem Urin, der viele verwandt reagierende Stoffe enthält.

Von dẹn heute zur Verfuigung stehenden Analysenmethoden genügen diesen Ansprüchen am besten die Gaschromatographie-Massenspektrometrie (GC-MS)-Kopplung $(1,2)$ und radioenzymatische Verfahren $(3,12)$. Deren geringe Verbreitung dürfte sowohl auf den hohen finanziellen und apparativen Aufwand als auch auf die Forderung nach bes̄onders versiertem Laborpersonal zurückzuführen sein.

Praktikable Alternativen sind zu erhoffen von der Hochdruckflüssigchromatographie mit fluorimetrischer Detektion und der Kapillar-Gaschromatographie in Verbindung mit einem Elek troneneinfangdetëktor. Für die fluoreszierenden Dansyl-, Trihydroxyindol-, o-Phthaldialdehydund Fluorescaminderivate liegen gründliche Studien vor $(4,5,6,7)$, keines dieser Verfahren ist bisher für die klinisch-chemische Routine genügend ausgereift. Die Dansylderivate sind zersetzlich; die TrihydroxyindolReaktion erfordert einigen technischen Aufwand; 0 Phthaldialdehyd und Fluorescamin reagieren nur mit primären Aminen. Mit Ausnahme der Arbeit von Imai (8), werden die angegebenen Verfahren zudem nicht auf physiologische Flüssigkeiten angewandt.

Die gaschromatographische Bestimmung der Pentafluorpropionyl-Derivate der Amine erwies sich in unserem Labor als höchst problematisch: Entweder erreichten wir mit gepackten Säulen keine ausreichende Trennung oder die Derivate zersetzten sich in der Glaskapillare.

Demgegenüber ist die Technik der automatisierten Aminosäurechromatographie seit Jahren ausgereift. In der von uns hier vorgelegten Methode wird die quantitative Analyse der Summe aus freiem und konjugiertem Noradrenalin, Dopamin und Normetanephrin aus dem Urin mit einem Aminosäureanalysator beschrieben. Einen ähnlichen Weg sind Wall (9); Cronin (10) und Villanueva (11) bei der Bestimmung einiger Amine aus pflanzlichen Extrakten, extraterrestischem Material und Standardlösungen gegangen. 


\section{Methodik}

Der Analysator setzte sich aus folgenden Bausteinen zusammen:

Programmiereinheit BT 5052 (Biotronik)

2 Dosapro-Pumpen 19633 (Milton-Roy)

Ultrathermostat K 5 (Colora)

Schreiber PM 8000 (Philips)

Fluorimeter BT 6630 (Biotronik)

Austauschersäule $6 \mathrm{~mm}$ i.D. (Biotronik)

Austauscherharz DC 6 A (Durrum)

Integrator Autolab System I (Spectra-Physics)

Zur Pulsationsdämpfung wurde zwischen dem Rückschlagventil der Reagenzpumpe und Mischblock ein Teflonschlauch (30 $\mathrm{m} \times 0,3 \mathrm{~mm})$

eingesetzt.

\section{Elutionspuffer}

21,0 g/1 Lithiumchlorid (Merck, Nr. 5679) und 31,5 g/1 Lithiumcitrat (Merck, Nr. 5683); der pH-Wert von 3,95 wurde mit Lithiumhydroxid (Merck, Nr. 5691) und Salzsäure' (Merck, Nr. 319) eingestellt.

\section{Probenverdünnungspuffer}

$14,0 \mathrm{~g} / 1$ Lithiumcitrat; $\mathrm{pH}=3,50$.

\section{Reaktionslösung}

11 einer Borsäurelösung $(81,63 \mathrm{~g} / 1$, Merck, Nr. 765$)$ wird mit $\mathrm{KOH}(35 \mathrm{~g} / \mathrm{l})$ auf $\mathrm{pH}=10,30$ gebracht. Dazu gibt man tropfenweise $3 \mathrm{ml}$ Mercaptoethanol (Merck, Nr. 805740) und $10 \mathrm{ml}$ einer ethanolischen $o$-Phthaldialdehydlösung $(50 \mathrm{~g} / \mathrm{l}$, Merck, Nr. 11452). Anschließend wird die Lösung durch Anlegen eines Wasserstrahlvakuums gründlich entlüftet und danach $2 \mathrm{ml}$ einer Brij-35-Lösung (300 g/l; Fluka 16005) zugegeben. Die Reaktionsfähigkeit dieser Lösung bleibt etwa eine Woche erhalten. (Mercaptoethanol sollte bei $4^{\circ} \mathrm{C}$ unter Stickstoff aufbewahrt werden).

\section{Elutionsbedingungen}

Puffer- und Reagenzdurchfluß: $52 \mathrm{ml} / \mathrm{h}$

Säulentemperatur: $65^{\circ} \mathrm{C}$

Füllhöhe: $85-90 \mathrm{~mm}$

Reaktionscoil: Länge $15 \mathrm{~m}, 0,3 \mathrm{~mm}$ i. D.; die Reaktion erfolgt bei Raumtemperatur.

\section{Probenvorbereitung}

$20 \mathrm{ml}$ Urin werden mit konzentrierter Salzsäure auf pH 0,75 gebracht und zentrifugiert. $5 \mathrm{ml}$ des Überstandes werden im Wasserbad bei $100^{\circ} \mathrm{C}$ während $20 \mathrm{~min}$ hydrolysiert. Nach dem Abkühlen auf Raumtemperatur werden $14 \mathrm{ml}$ einer DiNatrium-ethylen-di-nitrolo-tetraessigsäure-dihydrat (EDTA-, Titriplex -III-)Lösung hinzugegeben $(3,36 \mathrm{~g} / \mathrm{l})$ und $\mathrm{mit} \mathrm{NaOH}$ $(300 \mathrm{~g} / \mathrm{l})$ auf pH 6,50 gebracht. Diese Lösung wird auf eine BioRad Fertigsäule gegeben. (Diese Säulen - schwach saure Kationenaustauscher in Na-Form - sind Bestandteile des Catecholamin-Kits der Firma Bio-Rad, München). Nach Waschen der Säule mit 22,5 $\mathrm{ml} \mathrm{H}_{2} \mathrm{O}$ werden Noradrenalin und Dopamin

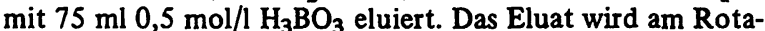
tionsverdampfer bei $35^{\circ} \mathrm{C}$ bis zur Trockene eingedampft und in $3 \mathrm{ml}$ des Probenverdünnungspuffers aufgenommen. 0,4 ml dieser Lösung und $0,02 \mathrm{ml}$ der Normetanephrinstandardlösung $(0,019 \mathrm{~g} / \mathrm{l})$ werden injiziert.

Die gleiche Säule wird dann mit 7,5 $\mathrm{ml} \mathrm{H}_{2} \mathrm{O}$ gewaschen und Normetanephrin mit 7,5 $\mathrm{ml} \mathrm{NH}_{4} \mathrm{OH}(4 \mathrm{~mol} / \mathrm{l})$ eluiert. Das Eluat wird bei $\mathrm{pH}=11,0$ dreimal während $3 \mathrm{~min}$ mit je $40 \mathrm{ml}$ Essigsäureethylester extrahiert. Die vereinigten Extrakte werden am Rotationsverdampfer bei $35^{\circ} \mathrm{C}$ zur. Trockene eingedampft. Nach Aufnahme des Rückstandes in $1 \mathrm{ml}$ Probenverdünnungspuffer werden $0,4 \mathrm{ml}$ der erhaltenen Lösung injiziert.

\section{Ergebnisse}

Abbildung 1 zeigt das Elutionsdiagramm einer Standardprobe, die 302 ng Noradrenalin, 364 ng Normetanephrin und $606 \mathrm{ng}$ Dopamin enthält. In Abbildung 2 und Abbildung 3 sind die Chromatogramme des Borsäure- bzw. des Ammoniumhydroxideluats eines Normalharns dargestellt. In Tabelle 1 sind die Qualitätskriterien der beschriebenen Methode angegeben.

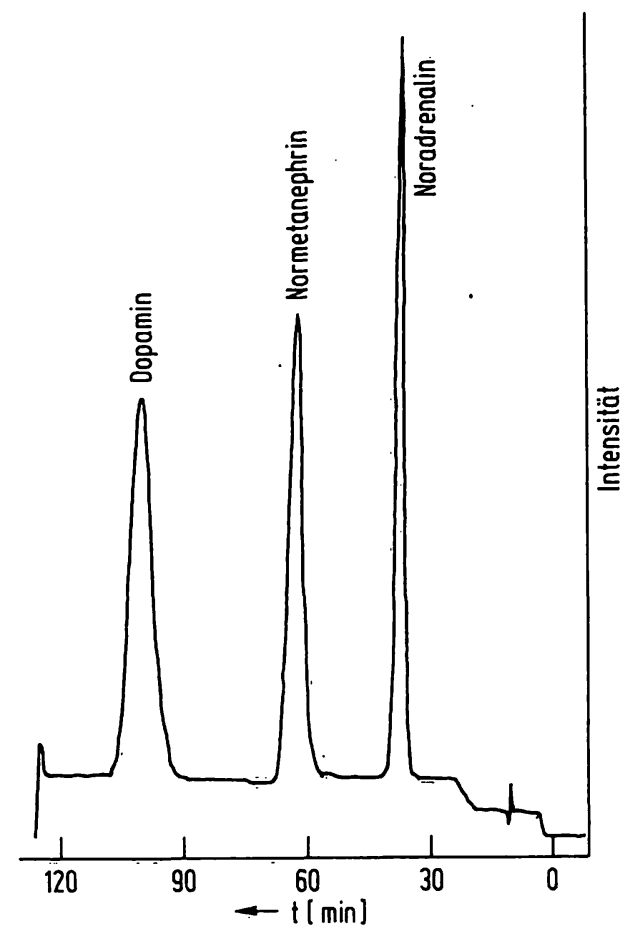

Abb. 1. Chromatogramm einer Standardlösung; Bedingungen siehe Text, Detektor Stufe 10.

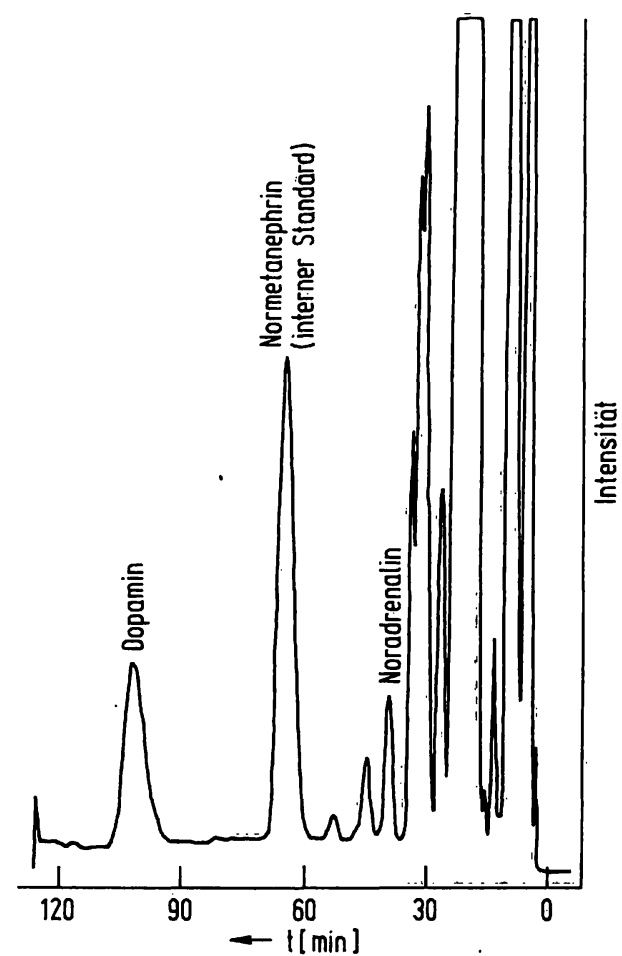

Abb. 2. Chromatogramm des Borsäureeluats der Bio.Rad-Fertiğsäule mit Dopamin und Noradrenalin; (Normalharn ohne Zusatz von Standardsubstanzen.) Bedingungen siehe Text; Detektor - Stufe 10. 


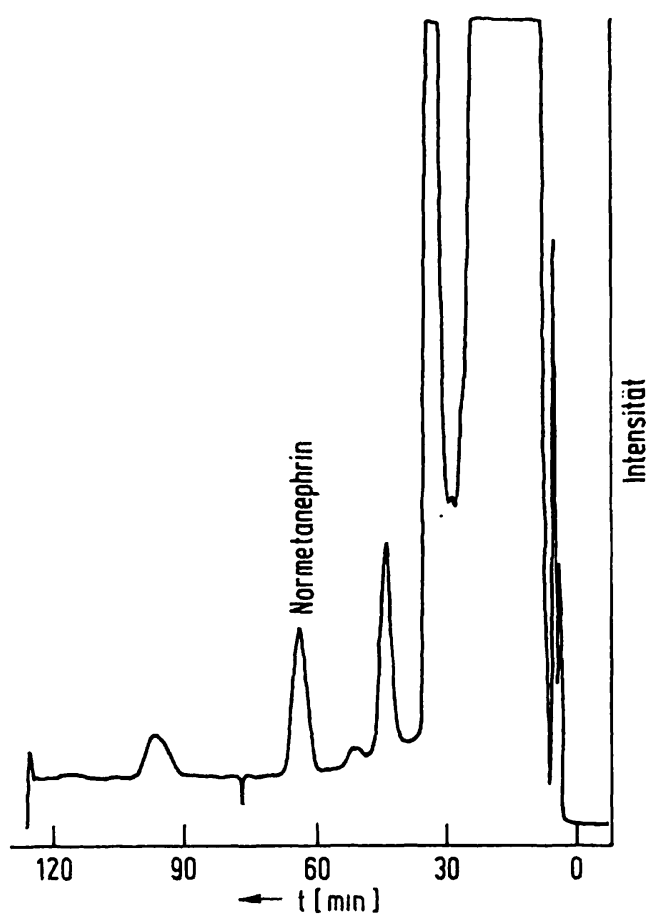

Abb. 3. Chromatogramm des Ammoniumhydroxideluats der Bio Rad-Fertigsäule mit Normetanephrin; (Normalharn ohne Zusatz von Standardsubstanzen.) Bedingungen siehe Text; Detek tor - Stufe 10.

Tab. 1. Qualitätskriterien der Bestimmung von Noradrenalin, Dopamin und Normetanephrin. Die angegebenen Werte wurden für den gesamten Analysengang ermittelt.

\begin{tabular}{|c|c|c|c|c|}
\hline Substanz & $\begin{array}{l}\text { überprüfter } \\
\text { Linearitäts- } \\
\text { bereich } \\
\text { ng/1 }\end{array}$ & $\begin{array}{l}\text { Richtig- } \\
\text { keit } \\
(\%) \\
n=10\end{array}$ & $\begin{array}{l}\text { Präzi- } \\
\text { sion } \\
\text { VK }(\%)\end{array}$ & $\begin{array}{l}\text { Nachweis- } \\
\text { grenze } \\
\text { (ng) }\end{array}$ \\
\hline $\begin{array}{l}\text { Normetanephrin } \\
\text { Noradrenalin } \\
\text { Dopamin }\end{array}$ & $\begin{array}{r}50-750 \\
37,5-375 \\
250-2500\end{array}$ & $\begin{array}{l}53,4 \\
67,8 \\
83,3\end{array}$ & $\begin{array}{l}2,6 \\
2,8 \\
5,3\end{array}$ & $\begin{array}{r}4 \\
1 \\
15\end{array}$ \\
\hline
\end{tabular}

Richtigkeit und Präzision wurden für den gesamten Analysengang ermittelt, d. h. der durch die Säurehydrolyse bedingte Fehler wurde berücksichtigt.

Zur Ermittlung der Präzision in der Serie wurde eine Urinprobe 10mal aufgearbeitet. Die hierbei erhaltenen Variationskoeffizienten sind in Tabelle 1 angegeben. Anschließend wurde zu je $5 \mathrm{ml}$ der gleichen Ürinprobe zwischen $500 \mathrm{ng}$ und $1358 \mathrm{ng}$ Normetanephrin, $790 \mathrm{ng}$ und 1400 ng Noradrënalin bzw. 1300 ng und 2600 ng Dopamin zugese tzt. Mit Hilfe unserer Standardkurve ermittelten wir die diesen zugeführten Substanzmengen entsprechenden Flächenausbeuten. In unserer Bestimmung der Richtigkeit wurden diese Werte gleich $100 \%$ gesetzt. Die Differenz aus Normalurinkonzentrationen plus zugese tztem Standard und Normalurinkonzentration ohne Zusatz ergab dann den in Tabelle 1 angege- benen Wert für die Richtigkeit. Letztere blieb für alle getesteten Konzentrationen gleich. Die Nachweisgrenze entspricht dem doppelten Wert des Grundrauschens unter den angeführten Analysenbedingungen.

Auffallen mag, daß bei Standardlösungen Noradrenalin, Dopamin und Normetanephrin in einem Chromatogramm bestimmt werden, während in Urinproben Normetanephrin getrennt analysiert wird. Dieses Vorgehen erschien uns günstiger, da die Noradrenalin und Dopamin enthaltende Urinfraktion soviel Borsäure enthält, daß $3 \mathrm{ml}$ Probenverdünnungspuffer zum Lösen nötig sind. Die Normetanephrin en thaltende Urinfraktion kann jedoch bequem in $1 \mathrm{ml}$ Probenverdünnungspuffer aufgenommen werden, wodurch eine günstigere Nachweisgrenze erzielt wird. Darüber hinaus ist es jetzt möglich, jeder zweiten Analyse Normetanephrin alsexternen Standard zuzusetzen, und so das Nachlassen der Reaktionsbereitschaft der Reagenzlösung auszugleichen.

Im Gegensatz zu den Angaben von da Prada (12) erreichen wir für Normetanephrin mit Essigsäureethylester eine bessere Extraktionsausbeute im Vergleich zur Extraktion mit Diethylether unter Zusatz von Natriumtetraphenylborat.

Tabelle 2 gibt die Mittelwerte der im 24 h-Urin von 9 Kontrollpersonen gefundenen Konzentrationen an Noradrenalin, Dopamin und Normetanephrin wieder.

Die erhaltenen Werte für Noradrenalin und Normetanephrin stimmen mit den in der Literatur angegebenen Konzentrationen überein (13-16). Die gemessene Dopaminkonzentration beträgt jedoch ungefähr das sechsfache mancher Literaturangaben. Dies mag darauf zurückzuführen sein, daß die Ausscheidung von Dopamin stark von der Ernährung abhängt.

Die weite Verbreitung von automatischen Aminosäureanalysatoren in klinisch-chemischen Labors erschließt der beschriebenen Methode eine breite Anwendung. Die Umrüstung eines Standard-Aminosäureanalysators auf die $o$-Phthaldialdehyd-Reaktion, auch zur Bestimmung der Aminosäuren, bietet einige Vorteile: Bei gesteigerter Nachweisempfindlichkeit wird eine ruhigere Basislinie und ein größerer Linearitätsbereich erzielt, die Reagenzlösung benötigt weniger Schutzvorkehrungen zum Erhalt ihrer Reaktionsfähigkeit und sie ist um ungefähr $50 \%$ billiger als eine Ninhydrinlösung.

Tab. 2. Mittelwert der Konzentration an Noradrenalin, Dopamin und Normetanephrin im Urin von 9 Kontrollpersonen.

\begin{tabular}{lcl}
\hline Substanz & $\begin{array}{l}\overline{\mathrm{x}} \pm \mathrm{s} \\
(\mu \mathrm{g} / 24 \mathrm{~h})\end{array}$ & $\begin{array}{l}\text { Bereich } \\
(\mu \mathrm{g} / 24 \mathrm{~h})\end{array}$ \\
\hline Noradrenalin & $165,2 \pm 60,9$ & $\begin{array}{l}110,2-315,27 \\
\text { Dopamin }\end{array}$ \\
$\begin{array}{l}1473,5 \pm 678,5 \\
\text { Normetanephrin }\end{array}$ & $\begin{array}{l}760,9-2856,9 \\
270,9 \pm 37,5\end{array}$ & $219,5-326,9$ \\
\hline
\end{tabular}




\section{Literatur}

1. Robertson, D., Heath, E. C., Falkner, F. C., Rayburn, E. H., Brilis, G. M. \& Watson, J. T. (1978), Biomed Mass Spectrometry 5, 704-708.

2. Ehrhardt, J. D. \& Schwartz; J. (1978), Clin. Chim. Acta 88, 71-79.

3. Engelmann, K., Portnay, B. \& Lovenberg, W. (1968), Am. J. Med. Sci. 255, 259-268.

4. Schwedt, G. (1977), Anal. Chim. Acta 92, 337-344.

5. Schwedt, G. (1976), J. Chromatogr. 118, 429-432.

6. Schwedt, G. \& Bussemas, H. H. (1978), Z. Anal. Chem. 283, 23-28.

7. Schwedt, G. (1977), J. Chromatogr. 143, 463-471.

8. Imai, K. \& Tamura, Z. (1978), Clin. Chim. Acta 85, 1-6.

9. Wall, R. A. (1971), J. Chromatogr. 60, 195-202.

10. Cronin, J. R. \& Hare, P. E. (1976), Anal. Biochem. 81, $151-156$.
11. Villanueva, V. R., Adlakha, R. C. \& Cantera-Soler, A. M. (1977), J. Chromatogr. 139, 381-385.

12. Da Prada, M. \& Zürcher, G. (1976), Life Science 19, 1161-1174.

13. Nelson, L. M., Bubbb, F. A., Lax, P. M. \& Sandler, M. (1979), Clin. Chim. Acta 92, 235-240.

14. Robertson, D., Fröhlich, J. C., Carr, R. K., Watson, J. T., Hollifield, J. W., Shand, D. G. \& Cates, J. A. (1978), N. Engl. J. Med. 298, 181 .

15. Takahashi, S., Leiv, R. \& Gjessing, D. (1972), Clin. Chim. Acta 36, 369-378.

16. Winkel, P. \& Slob, A. (1973), Clin. Chim. Acta 45, 113-118.
Dr. Klaus Olek

Institut für Humangenetik der Universität Bonn Wilhelmstraße 31

D-5300 Bonn 